\title{
Correction to: Denosumab does not decrease the risk of lung metastases from bone giant cell tumour
}

\author{
Shinji Tsukamoto ${ }^{1}$. Andreas F. Mavrogenis ${ }^{2}$ (D) Giulio Leone $^{3}$ - Alberto Righi ${ }^{4}$. Manabu Akahane ${ }^{5}$. \\ Piergiuseppe Tanzi ${ }^{6} \cdot$ Akira Kido $^{1} \cdot$ Kanya Honoki $^{1} \cdot$ Yasuhito Tanaka $^{1} \cdot$ Davide Maria Donati $^{6} \cdot$ Costantino Errani $^{6}$
}

Published online: 21 September 2018

(C) SICOT aisbl 2018

\section{Correction to: International Orthopaedics https://doi.org/10.1007/s00264-018-4085-6}

Last and second name of an author have been mixed up. It shows Piergiuseppe as the last name, but his second name is Tanzi and the first name is Piergiuseppe.

The original article has been corrected.

The online version of the original article can be found at https:/doi.org/ 10.1007/s00264-018-4085-6

Shinji Tsukamoto

shinji104@mail.goo.ne.jp

$\triangle$ Andreas F. Mavrogenis

afm@otenet.gr

$\triangle$ Costantino Errani

costantino.errani@ior.it

1 Department of Orthopaedics, Nara Medical University, Nara, Japan

2 First Department of Orthopaedics, National and Kapodistrian University of Athens, School of Medicine, 41 Ventouri Str, 15562 Athens, Greece

3 Department of Orthopaedics, San Gerardo Hospital, Monza, Italy

4 Department of Pathology, Istituto Ortopedico Rizzoli, Bologna, Italy

5 Department of Public Health, Health Management, and Policy, Nara Medical University, Nara, Japan

6 Department of Orthopaedic Oncology, Istituto Ortopedico Rizzoli, Bologna, Italy 